\title{
Advanced model atmospheres with magnetic field effects included
}

\author{
Denis Shulyak ${ }^{1}$, S. Khan ${ }^{2}$, and O. Kochukhov ${ }^{3}$ \\ ${ }^{1}$ Institute for Astronomy, Vienna University, Türkenschanzstrasse, 17, 1180 Vienna, Austria \\ email: denis@jan.astro.univie.ac.at \\ ${ }^{2}$ Institute for Computational Astrophysics, Saint Mary's University, 923 Robie Street, Halifax, \\ B3H 3C3, Nova Scotia, Canada \\ email: serg@starsp.org \\ ${ }^{3}$ Department of Astronomy and Space Physics, Uppsala University, Box 515, 75120 , \\ Uppsala, Sweden \\ email: Oleg.Kochukhov@fysast.uu.se
}

\begin{abstract}
The atmospheres of magnetic chemically peculiar (mCP) stars display the presence of magnetic fields of different geometry and strength, ranging from a few hundred $\mathrm{G}$ up to tens of $\mathrm{kG}$. Except several very approximate attempts there were no detailed studies of magnetic field effects on model atmospheres structure, possibly leading to errors in the stellar parameter determination and abundance analysis routines. We present the magnetic model atmospheres based on LLmodels code which accounts for the detailed treatment of anomalous Zeeman splitting and polarized radiative transfer.
\end{abstract}

Keywords. Stars: atmospheres - stars: magnetic fields - stars: chemically peculiar

\section{Introduction}

The atmospheres of magnetic chemically peculiar $(\mathrm{mCP})$ stars exhibit the presence of large-scale magnetic fields that can have the strength about tens of $\mathrm{kG}$. However, the magnetic field effects are ignored in standard-model atmosphere calculations. To circumvent this longstanding problem, we have developed a new model atmosphere code LLMODELS (Shulyak et al. 2004) which account for detailed treatment of anomalous Zeeman splitting (Kochukhov et al. 2005), polarized radiative transfer (Khan \& Shulyak 2006a) and inclination of the magnetic field vector (Khan \& Shulyak 2006b).

\section{Model predictions}

We have computed a grid of model atmospheres and investigated the effect of magnetic field on model structure, energy distribution, hydrogen line profiles, and photometric colors. The main results can be summarized as follows:

- Magnetic opacity leads to the heating of certain atmospheric layers.

- Flux redistribution from UV to visual region with the increase of the magnetic field strength.

- Flux depression at $5200 \AA$ appears for lower temperatures and vanishes for the higher ones for a given magnetic field modulus.

- Relation between photometric indexes and the magnetic field strength is not linear due to the saturation effect (except $\Delta a$ index for cool models) and depends strongly upon the metallicity of the models assumed. 
Table 1. Observed and calculated photometric parameters of HD 137509. Suffixes "m", "n" and "s" refer to magnetic, non-magnetic (both with individual abundances), and non-magnetic scaled-solar abundance model from Kochukhov (2006). The improved models are presented in last two rows.

\begin{tabular}{l|cccccc}
\hline & $b-y$ & $c_{1}$ & $\Delta a$ & $X$ & $Y$ & $Z$ \\
\hline observations & $-\mathbf{0 . 0 9 5}$ & $\mathbf{0 . 4 1 1}$ & $\mathbf{0 . 0 6 6}$ & $\mathbf{0 . 7 6 2}$ & $\mathbf{0 . 0 7 6}$ & $\mathbf{- 0 . 0 6 7}$ \\
\hline t12750g3.8m & -0.056 & 0.573 & 0.054 & 0.936 & 0.107 & -0.043 \\
t12750g3.8n & -0.043 & 0.588 & 0.032 & 0.957 & 0.066 & -0.028 \\
t12750g3.8s & -0.026 & 0.581 & 0.014 & 0.942 & 0.032 & -0.016 \\
\hline t13750g4.2m & -0.081 & 0.453 & 0.062 & 0.799 & 0.081 & -0.051 \\
t13750g4.2n & -0.064 & 0.470 & 0.037 & 0.818 & 0.040 & -0.036 \\
\hline
\end{tabular}

- The $\Delta a$ system is not able to clearly distinguish CP stars with metallicity other than solar for low $T_{\text {eff }}$, thought it remains the most sensitive one to the field strength among the others for hotter stars.

- Magnetic opacity does not introduce significant errors in the photometric estimates of CP-stars parameters.

- The effect of magnetic opacity on model structure do not influence much the hydrogen lines profiles.

- The widely used models computed with pseudo-microturbulence parameter to simulate the magnetic field effects can only be used as a very rough guess.

- The inclination of the magnetic field vector has small impact on model atmosphere structure and energy distribution.

\section{Strong magnetic field star HD 137509}

HD137509 is a B-type star with the second-largest magnetic field ever found among CP stars with $B_{\mathrm{z}}=29 \mathrm{kG}$, which is the excellent test-ground for newly developed magnetic models. The fundamental parameters $T_{\text {eff }}=12750 \mathrm{~K}, \log g=3.8$ were previously derived using standard non-magnetic model computed with $[M / H]=+1$ (Kochukhov 2006). To improve the fundamental parameters of the star in a consistent way we redetermined the abundances using model atmospheres computed with LLMODELS with both magnetic field and individual abundances taken into account. Some photometric colors of models computed with initial abundances from Kochukhov (2006) and models with improved fundamental parameters and abundances are presented in Table 1. We find that the simultaneous fit to the $\mathrm{H}$-line profiles and photometric indexes required as much as $1000 \mathrm{~K}$ correction to the $T_{\text {eff }}$ and 0.4 dex correction to the $\log g$ compared to the results obtained using simple scaled-solar models (for more details, see Shulyak et al. 2008).

\section{Acknowledgements}

This work was supported by FWF Lisa Meitner grant Nr. M998-N16 to DS.

\section{References}

Khan, S. \& Shulyak, D. 2006b, A\&\&A 454, 933

Khan, S. \& Shulyak, D. 2006a, A\&A 448, 1153

Kochukhov, O. 2006, A\& A 454, 321

Kochukhov, O., Khan, S., \& Shulyak, D. 2005, A\& A 433, 671

Shulyak, D., Kochukhov, O., \& Khan, S. 2008, A\&A 487, 689

Shulyak, D., Tsymbal, V., Ryabchikova, T., Stütz, Ch., \& Weiss, W. W. 2004, A\&A 428, 993 